\title{
Rheology of dense granular chute flow: simulations to experiments
}

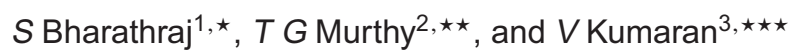 \\ ${ }^{1}$ Chemical Engineering, Indian Institute of Science, Bangalore, India \\ ${ }^{2}$ Civil Engineering, Indian Institute of Science, Bangalore, India \\ ${ }^{3}$ Chemical Engineering, Indian Institute of Science, Bangalore, India
}

\begin{abstract}
Granular chute flow simulations reveal an interesting transition from a random disordered structure to an ordered one with hexagonally ordered sheets of spherical particles, when the base roughness is modulated. Two types of base roughness are considered. The first is a fixed base, where glued spherical particles form the base, and the base roughness is varied by changing the ratio of diameters of the base and flowing particles. In the second sinusoidal base, a smooth wall with sinusoidal height variation is used; the amplitude and wavelength of the base modulation determine the base roughness. The transition is studied as a function of these roughness parameters. For the fixed base, there is a critical base particle diameter below which ordered states are observed. For the sinusoidal base, the critical amplitude increases linearly with the wavelength at lower wavelengths, reaches a maximum depending on the height of the flowing layer, and then decreases as the wavelength is further increased. There is flow for angles of inclination from $15^{\circ} \leq \theta \leq 25^{\circ}$ for the ordered state and $20^{\circ} \leq \theta \leq$ $25^{\circ}$ for the disordered state. Flow confinement by sidewalls also influences the rheology of the system and we see that the ordering is induced by the sidewalls as well. Experiments on chute flow at low angles indicate the presence of two types of rheology depending on the system height. A transition is observed from an erodible base configuration, where a dead zone at the bottom supports a free surface reposing at the top, to a Bagnold rheology with considerable slip at the bottom.
\end{abstract}

\section{Introduction}

Granular chute flow has been extensively used as a phenomenological model for understanding the mechanics of landslides, and industrial processes such as flow over a conveyor belt. Discrete element simulations have provided extraordinary insight into the mechanics of chute flow from the inter particle interactions through the overall flow profile. Although the results are quite sensitive to the input i.e. the model and parameters, several investigations over the years have provided a firm theoretical underpinning for this problem.

These simulations on chute flow have shown that the base configuration plays a pivotal role in the rheology of the granular ensemble and have also been verified through experiments [1][2]. The arrangement of the base in the simulations can broadly be divided into three categories, a bumpy base made of glued particles, an erodible base with movable or unconstrained particles and a smooth base with no particles but a smooth wall interacting with the flowing particles. In case of a bumpy base, the glued particles at the base are arranged with a random or an ordered structure, and the size of the glued base particles are also varied relative to the flowing ones. All these modulations effectively change the base roughness in the system. The

\footnotetext{
^e-mail: sagarbharathraj@gmail.com

$\star \star$ e-mail: tejas@civil.iisc.ernet.in

$\star \star \star$ e-mail: kumaran@chemeng.iisc.ernet.in
}

methodology of constructing the bumpy base has been explained in detail by Silbert [3], Jenkins and Richman [4]. Silbert reported the effect of the base configuration on the rheology, where hexagonally ordered glued particle bases resulted in ordering of the flowing particles, which organized into non-interacting layers [5]. Transition was also observed between states as a function of the base particle diameter.

The effect of randomly arranged fixed bases on the flow dynamics was reported by Kumaran and Maheshwari [6] for a linear contact model. A sharp discontinuous transition from a random disordered structure to one with hexagonally ordered layers of flowing sheets of particles was observed, when the fixed base particle diameter decreased from 0.62 to 0.61 , relative to the flowing particles (of diameter 1). These observations were independent of the angle of inclination and system depth. The ordered states exhibit steady flow for a chute angle, $\theta$, between $15<\theta<25$ and the disordered states flow between $20<\theta<25$. Both these states conform to the Bagnold rheology where the stress was proportional to the strain rate squared, (it should be noted here, that this is a dimensional necessity for a system with instantaneous contacts, as the inverse of strainrate presents the only relevant time scale in the system). The ordered state was found to have significantly higher mean and fluctuating velocities. The Bagnold coefficients are an order of magnitude higher for 
the ordered state due to lower strainrates. The volume density was also slightly higher for the ordered state due to better confinement. The number density and volume density profiles also show nice crests and troughs spaced by a particle diameter, indicative of the layering in the system. The ordering is quantified through the $2 \mathrm{D}$ order parameter $q_{6}$, which is defined as follows:

$$
q_{m}=\frac{1}{N} \sum_{i=1}^{N} \sum_{j \neq i} \exp \left(i m \theta_{i j}\right) .
$$

where $N$ is the total number of particles in the system and $m$ is 6 to check for a hexagonally ordered system. Thus, $q_{6}$ is 1 for a hexagonally ordered system and close to 0 for a disordered system. The evolution of these two states was studied by Kumaran and Bharathraj [7], who proposed a boundary layer formulation for the development of the ordered state. It was observed that the ordered state, during its evolution, has two distinct stages and has two distinct regimes during the first stage in the system. In the first stage, there is propagation of shear from the bottom and the shear front also carries with it the order front, which supports a disordered plug flow above it with no internal shear. The velocity in the shear region was found to obey the Bagnold law and the maximum velocity increases linearly with time in this first stage. When the shear and order simultaneously encompass the entire system, there is much slower increase in the maximum velocity, which is the second stage of development. The rate of propagation of this front was found to be dependent only on the angle of inclination of the chute. In contrast, the evolution of the disordered state showed the presence of shear stresses throughout the system [7]. It was also shown that the transition was observed for the Hertzian contact model [8]. The energy balance in the system reveals that the energy due to shear was balanced by the dissipation due to inelastic collisions except at the bottom and top layers, where there is a zone of conduction approximately up to 5 particle diameters. One consequence of the same is the scaling of the granular temperature as the square of the strainrate.

Extensive experiments on granular rheology have revealed very interesting physics of various flow instabilities, and regimes. We present a series of experiments that were carried out on a glass chute, using glass beads for small chute angles. We further present in this paper a series of simulations examining the effect of another type of base modulation, wherein a smooth wall at the bottom is provided a periodic structure of the form, $w_{0}+$ $a \cdot \sin (2 \pi p / \lambda)$, where $\omega_{0}$ is the reference position of the wall, $a$ is the amplitude of the position perturbation, $\lambda$ is the wavelength, $p$ is either the flow direction $x$, or the span direction, $y$. We report the transition as a function of the wavelength, amplitude and direction of the perturbation. We specifically seek to highlight the variation in the rheology for such a smooth wall problem from that of a fixed base configuration. The evolution of the ordered and disordered states induced by these different base modulations in addition, to preliminary observations on flow confinement in the presence of side walls are also presented here [9].

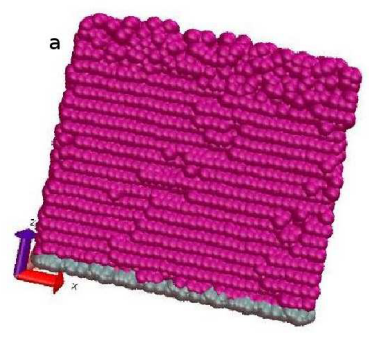

$$
\begin{aligned}
& d_{b}=0.58 \\
& d_{f}=1
\end{aligned}
$$

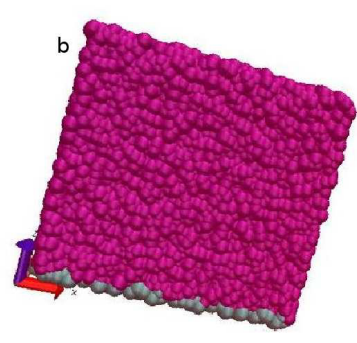

$\mathrm{d}_{\mathrm{b}}=\mathbf{0 . 6 0}$

$d_{\mathrm{f}}=1$
$d_{\mathrm{f}}=1$
Figure 1. Snapshot of (a) an ordered and (b) a disordered system with flowing particles of diameter, $d_{f}$ and a bumpy base with glued particles of diameter, $d_{b}$

\section{Experimental and Simulation methodology}

The simulation cell consisted of monodisperse spherical particles stacked up in all the three dimensions, with periodic boundary conditions in the flow $(x)$ and $\operatorname{span}(y)$ directions. The flow is confined by a base at the bottom and a free surface at the top as discussed in the previous section. LAMMPS, an open source molecular dynamics package, implementing Newtonian mechanics for individual particles with inter-particle contact laws, is used for simulations with the Hertzian inter-particle contact model. All the relevant quantitites in the system are non dimensionalised with suitable system parameters. All masses and lengths are scaled by the mass $m$ and diameter $d$ of the flowing particles, and all times by $\sqrt{d / g}$. Details of the contact model and its implementation are available in previous studies [3][6][7][8]. The important quantities monitored are the average velocity in the flow direction, $V_{x}$, the average coordination number, $Z$, the number and volume densities, $n, \phi$, the order parameter, $q_{6}$ were all computed from these simulations and are compared to experiments for certain conditions. The averaging techniques used to calculate these parameters and the input simulation parameters is available [6][7].

A schematic of the ordered and disordered system is given in figure 1. Experiments were carried out on a glass chute with dimensions $150 \mathrm{~cm} \times 10 \mathrm{~cm} \times 10 \mathrm{~cm}$. Glass beads of diameter $2-2.5 \mathrm{~mm}$ were used as a model system for these experiments. Imaging was carried using a high frame rate camera(PCO 1200HS). The images obtained from the camera were analysed using PTVlab [14] and velocity fields are obtained after calibration.

\section{Results and discussions}

\subsection{Transition due to a periodic smooth base}

Transition from a random disordered structure to a hexagonally ordered one, occurs when different types of forcing (or modulations) are provided at the base. For the set of simulations performed on a rough bumpy base, this transition occurs at critical base particle diameter of 0.59 for the 

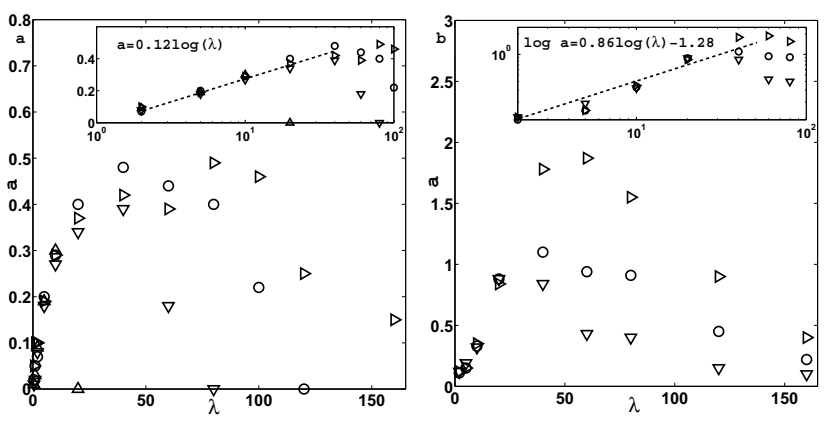

Figure 2. Transition amplitude, $a$, as a function of the wavelength, $\lambda$, of the bottom smooth wall for modulations in the : (a) flow direction, $x$, and (b) span direction, $y$, with the corresponding log curves in the inset with the model fit (- -), for different system heights, $z=18(\triangle), 25(\nabla), 37(\circ), 75(\triangleright)$ at an angle of inclination, $22^{\circ}$

Hertzian model. As the bumpy base has a random (irregular) structure, the base roughness is also aperiodic. This transition is studied as a function of the amplitude, wavelength and direction of the modulation, as shown in figure 2. As seen in the figure 2, the transition amplitude, $a$, increases linearly with wavelength, $\lambda$ at lower wavelengths irrespective of the system depth. The amplitude then reaches a critical value and then decreases with wavelength, depending on the system depth. As is evident from the figure, at a given wavelength, the transition amplitude is much larger for the span wise $(y)$ modulation than the flow wise $(x)$.

We further compare the velocity profiles of the ordered-disordered states for the sinusoidal base with the profiles obtained with the fixed base. For this, we plot the flow velocity, $v_{x}$, and the order parameter, $q_{6}$, profiles as a function of the depth, $z$, for the ordered and disordered states obtained with the two types of base modulations as shown in figure 3 . The average velocity profile in figure 3(a) reveals that all the configurations follow the Bagnold rheology, except for the bottom few layers of the disordered state generated by the sinusoidal base. A significant difference being the slip generated by the smooth wall in both these states, it is interesting to note that when this profile is subtracted from the bulk, we approximately recover the profiles of the fixed base states. The averaged order parameter, $q_{6}$, is approximately 1 in the bulk for the ordered states and 0 for the disordered states, as expected. However, in case of the sinusoidal bases, the profile reveals the presence of a few layers of disorder at the bottom, up to around 5 particle diameters, where the hexagonal order is imperfect. This feature in the ordered states of the smooth wall case is universal, and is found to be independent of the wavelength, amplitude and direction of the modulation.

Another feature, which is seen only in the smooth wall modulations, is the tendency of ordering in the disordered states, just above the critical transition amplitude. The order seems to propagate from the bottom to the bulk, but seems to be disrupted before it reaches the top surface. In
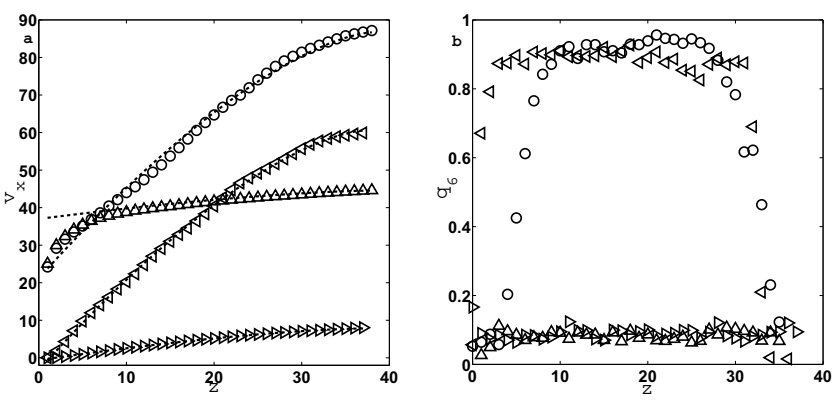

Figure 3. (a) Average flow velocity, $v_{x}$ with the Bagnold fit (- -), (b) averaged $2 \mathrm{D}$ order parameter, $q_{6}$ variation with system depth, $z$, for an ordered state generated by a sinusoidal smooth base (०), a rough base $(\triangleleft)$, and a disordered state generated by a sinusoidal smooth base $(\triangle)$, a rough base $(\triangleright)$, at an angle of inclination, $22^{\circ}$

the evolution of the ordered state, the propogation of the order front can be treated in a boundary layer formulation, wherein we define the height up to which the $q_{6}$ is 0.5 or higher, as the boundary layer thickness. It was reported that the order front has a universal slope of 1 particle diameter in 13.5 dimensionless timesteps, dependent only on the angle of inclination [7]. Here, we find the same observation even for sinusoidal bases, apart from the disordered bottom layers.

\subsection{Effect of confinement with sidewalls}

The effect of boundaries on the flow rheology has been well documented, as outlined earlier. We present a series of simulations on a system confined between sidewalls [10]. In figure 4, the effect of variations in the span on the flow velocity is presented. As seen in figure 4, a significant difference exists in the magnitude of the velocity at the wall and away from the wall. In case of the system with a total span of 20 particle diameters, a dead zone measuring up to 5 particle diameters exists at the bottom, and the average flow velocity is lower compared to a system with periodic boundaries. The overall flow profile is convex, indicating an erodible base configuration. For the remaining systems, we see that there is a significant slip at the bottom and a concave profile with height, indicative of the Bagnold rheology. The magnitudes of the velocity in the span and height directions is less than $1 \%$ of the flow direction magnitudes.

The profile variations with the spanwise co-ordinate, $y$, also show interesting features. The percolation effect of the sidewalls on the flow velocity does not seem to affect a small system (i.e. with total span of 20 particle diameters) and is reasonably evident at lower depths for the remaining systems. The presence of side walls in the simulations also increases the minimum angle of inclination required for steady flow, from $15^{\circ}$ to $30^{\circ}$ for a system with a span of 20 particle diameters. For the system confined by smooth sidewalls and a bottom wall, a 3D ordering was also observed. The effect of the system height, aspect ratio, different types of sidewalls, ordering effects are discussed elsewhere. 

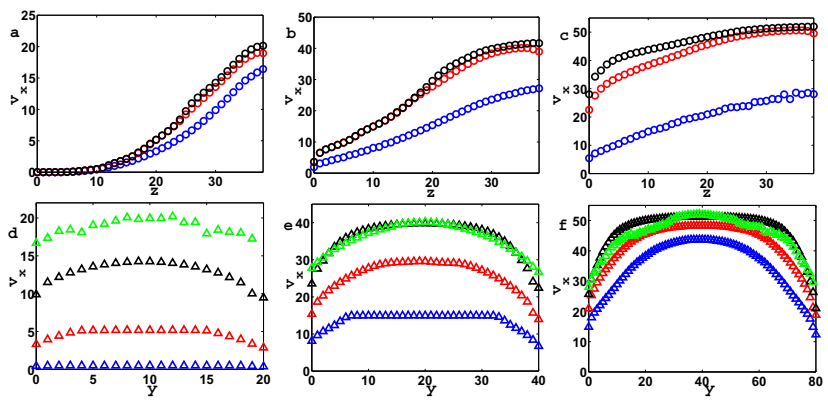

Figure 4. Average flow velocity, $v_{x}$ variation with height, $z$, and span, $y$, for a system with total span $=20$ (a)(d), 40(b)(e), 80(c)(f) at different locations; $y=$ wall $(\circ)$, centre $(\circ)$, in between wall and centre(o); z=10( $\triangle), \mathrm{z}=20(\triangle), \mathrm{z}=30(\Delta), \mathrm{z}=40(\triangle)$ for an angle of inclination, $30^{\circ}$
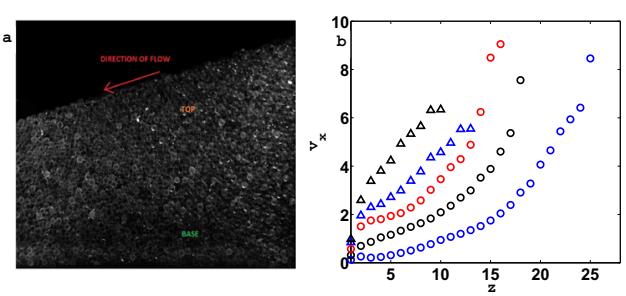

Figure 5. (a) Image obtained from the high frame rate camera of the glass beads flowing through the glass chute and (b) the variation of the steady flow velocity, $v_{x}$ with system height, $z$ for different system heights, $\mathrm{z}>25(\circ), 15<\mathrm{z}<20(\circ), \mathrm{z}=15(\circ), 10<$ $\mathrm{z}<15(\triangle), \mathrm{z}<10(\triangle)$ for an angle of inclination, $11^{\circ}$

\subsection{Experiments at low angles}

Experiments on granular chute flow have been performed mostly at intermediate and higher angles of inclinations [11] [12][13]. The rheology at lower angles, $9^{\circ}-16^{\circ}$ show some intriguing features. One such feature is the existence of regimes in the flow which depend on the total system height. Figure 5(b) clearly shows the change in the curvature of the velocity profile with the total system height. For a system height greater than 20 particle diameters, there is a prominent dead zone in the system, which extends to a height of 5 particle diameters and the velocity profile in the bulk is convex, which resembles the one obtained in figure 4(a). Below this height, the flow undergoes a transition as is evident in the figure. For systems with less than 15 particle diameters, the profile is concave adhering to the Bagnold rheology. It was observed that the free surface of the flow always occurs at the repose angle, irrespective of the chute angle, and is supported by a dead zone at the bottom. Once the flow height reduces below a critical value, the profile changes from an erodible convex one to a Bagnold concave one, which is also dependent on the slip velocity at the bottom.

\section{Conclusions}

The effect of different types of base modulations on the rheology is studied, which shows a transition from a random disordered state to one with hexagonally ordered 2D sheets, depending on the critical values of the base roughnesses. This transition is universal, irrespective of the contact model. The ordered and disordered states generated by the base modulations have many similarities and unique features. Flow confinement using sidewalls influences the rheology. The percolation effect of the sidewalls was observed as a function of different span depths. Experiments at very low angles indicate the existence of different flow regimes in the system, which seems to be influenced by the total height of flow and the slip at the bottom.

\section{References}

[1] Pierre Jop, Yoel Forterre, Olivier Pouliquen, Nature Letters 441, 727-30(2006)

[2] Olivier Pouliquen, Physics Of Fluids 11, 54248(1999)

[3] Leo Silbert, Deniz Ertaz, Gary S. Grest, Thomas C. Halsey, Dov Levine, Steven J. Plimpton, Physical Review E 64, 051302 1-14(2001)

[4] J.T Jenkins, M.W Richman, J. Fluid Mech 171, 5369(1986)

[5] Leornardo E Silbert, Gary S. Grest, Steven J. Plimpton, Physics Of Fluids 14, 2637-45(2002)

[6] V. Kumaran, S. Maheshwari, Physics Of Fluids 24, $0533021-23(2012)$

[7] V. Kumaran, S. Bharathraj, Physics Of Fluids 25, 070604 1-26(2013)

[8] P. A. Cundall, O. D. L. Strack, Geotechnique 29, 4765 (1979)

[9] J. Baker, T. Barker, J.M.N.T. Gray, J. Fluid. Mech 787, 367-395(2016)

[10] S. Bharathraj, V. Kumaran, (unpublished)

[11] Leornardo E Silbert, James W. Landry, Gary S. Grest, Physics Of Fluids 15, 1(2003)

[12] Thomas. G. Drake, J. Geophys. Res. 95, 86818696(1990)

[13] Emmanuel Azanza, Francois Chevoir, Pascal Moucheront, J. Fluid. Mech. 400, 199-227(1999)

[14] W. Brevis, Y. Nino, G.H Jirka, Experiments in Fluids, 50(1), 135-147(2011) 